\title{
Finding Gaussian Curvature of Lifespan Distribution
}

\author{
William W. S. Chen \\ Department of Statistics, The George Washington University, Washington DC, USA \\ Email: Williamwschen@gmail.com
}

Received 24 September 2014; revised 20 October 2014; accepted 2 November 2014

Copyright (C) 2014 by author and Scientific Research Publishing Inc.

This work is licensed under the Creative Commons Attribution International License (CC BY). http://creativecommons.org/licenses/by/4.0/

c) (i) Open Access

\begin{abstract}
The objective of this paper is to review the lifespan model. This paper will also suggest four additional general alternative computational methods not mentioned in Kass, R.E. and Vos, P.W. [1] [2]. It is not intended to compare the four formulas to be used in computing the Gaussian curvature. Four different formulas adopted from Struik, D.J. [3] are used and labeled here as (A), (B), (C), and (D). It has been found that all four of these formulas can compute the Gaussian curvature effectively and successfully. To avoid repetition, we only presented results from formulas (B) and (D). One can more easily check other results from formulas (A) and (C).
\end{abstract}

\section{Keywords}

Christoffel Symbols, Gamma, Gaussian Curvature, Inverse Gaussian, Metric Tensor, Mixed Riemann Curvature Tensor, Weibull

\section{Introduction}

The exponential, Weibull, gamma, lognormal, inverse Gaussian, and generalized gamma distributions are the most frequently used parametric lifespan models. Among the most commonly used lifespan models, the author has chosen three that he has studied since he was a graduate student. Lawless, J.F. [4] has suggested at least six different categories in applications. In the early 1980s, Chen, W. [5]-[8] pursued this area of study for two basic reasons. First, there was industrial interest. Engineering, medicine and biological sciences used the lifespan model to predict the best future values of their censored samples. Secondly, the author wanted to expand on his dissertation. If we summarized the last forty years of statistical research, the scientific community has had two fundamental topics to study. The first fundamental topic: suppose we are given a set of lifetime data, how do we decide which lifespan model best describes the data? A second interesting topic for researchers is data sets that can be "censored data”, where experiments in the sample only provide a lower or upper bound of lifetime. For 
example, Gupta, A. [9] presented results of a life-test on ten laboratory mice following inoculation with a uniform culture of human tuberculosis. The test was terminated with the death of the seventh specimen. Thus, the sample in this case was Type-II single right censored. Gupta then assumed that log lifespan was distributed normally with mean $\mu$ and variance $\sigma^{2}$, and then carried out the analysis as described in reference [10]. Chen, W. and Balakrishnan, N. [10] [11] have computed over one hundred thousand numerical integrations to find the moments of inverse Gaussian model and lognormal model, and then standardized these moments to find the best linear unbiased predicted sequences (EBLUP). Using these computed sequences it will give us the best prediction of the missing observations. However, in this paper we switch our attention to the geometrical property of lifespan model. To make it easier to follow what we have accomplished in this paper, we summarized our approach into four systematic steps to compute the Gaussian curvature: Step 1-compute the coefficients of the expected Fisher Information Matrix or coefficients of the first fundamental form, namely, $E, F$ and $G$; Step 2-compute the needed first or second derivative of $E, F$ and $G$, and thus the six Christoffel symbols; Step 3-apply formula (B) or (D), which necessitates in the computation of the mixed Riemann curvature tensors $\mathfrak{R}_{121}^{1}$ and $\mathfrak{R}_{121}^{2}$, then subsequent computation of the inner product of this tensor with the metric tensor, $F$ or $G$, results in the covariant Riemann curvature tensor $\Re_{1212}$, and Step 4-observe that the Gaussian curvature has a very simple relation to Riemann symbols of the second kind. By adhering to this procedure, the correct Gaussian curvature will be calculated. In the case where $F \neq 0$ or the parametric lines on the surface are not orthogonal, the computational procedure can be extremely tedious such as our model 2. It is always prudent to find a proper transformation to form an orthogonal system of parametric lines in order to simplify the computational procedures.

\section{Formulas}

In this section, we suggest four formulas that can be used to compute the Gaussian curvature.

$$
\begin{aligned}
& \text { (A) }-\frac{1}{\sqrt{E G}}\left(\frac{\partial}{\partial u}\left(\frac{1}{\sqrt{E}} \frac{\partial \sqrt{G}}{\partial u}\right)+\frac{\partial}{\partial v}\left(\frac{1}{\sqrt{G}} \frac{\partial \sqrt{E}}{\partial v}\right)\right) \\
& \text { (B) }-\frac{1}{4\left(E G-F^{2}\right)^{2}}\left|\begin{array}{ccc}
E & F & G \\
E u & F u & G u \\
E v & F v & G v
\end{array}\right|-\frac{1}{2 \sqrt{E G-F^{2}}}\left[\frac{\partial}{\partial u} \frac{G u-F v}{\sqrt{E G-F^{2}}}-\frac{\partial}{\partial v} \frac{F u-E v}{\sqrt{E G-F^{2}}}\right] \\
& \text { (C) } \frac{1}{D}\left[\frac{\partial}{\partial v}\left(\frac{D}{E} \Gamma_{11}^{2}\right)-\frac{\partial}{\partial u}\left(\frac{D}{E} \Gamma_{12}^{2}\right)\right]=\frac{1}{D}\left[\frac{\partial}{\partial u}\left(\frac{D}{G} \Gamma_{22}^{1}\right)-\frac{\partial}{\partial v}\left(\frac{D}{G} \Gamma_{12}^{1}\right)\right] \text { where } D^{2}=E G-F^{2} \\
& \text { (D) } \frac{\Re_{1212}}{E G-F^{2}}=\frac{(12,12)}{E G-F^{2}}, \text { where }(12,12)=\Re_{1212} \sum_{m=1}^{2} \Re_{121}^{m} g_{m 2}, \quad \Re_{i j k}^{l}=\frac{\partial}{\partial u_{j}} \Gamma_{i k}^{l}-\frac{\partial}{\partial u_{i}} \Gamma_{j k}^{l}+\Gamma_{i k}^{m} \Gamma_{m j}^{l}-\Gamma_{j k}^{m} \Gamma_{m i}^{l},
\end{aligned}
$$

sum on $m$, where the quantities of $\mathfrak{R}_{i j k}^{l}$ are components of a tensor of the fourth order. This tensor is called the mixed Riemann curvature tensor. Notice that $g_{11}, g_{12}$ and $g_{22}$ are simply tensor notation for $E, F$ and $G$. Formula (B) was developed by G. Frobenius while formula (C) was derived by J. Liouville. Clearly, formula (A) is a special case that is valid only when the parametric lines are orthogonal. Formula (D) is a general form represented in Riemann symbols of the first and second kind, respectively. In formula (D), $\mathfrak{R}_{1212}$, the inner product of the mixed Riemann curvature tensor and the metric tensor, is called the covariant Riemann curvature tensor; it is a covariant tensor of the fourth order. The components $\mathfrak{R}_{i j k}^{l}$ and $\mathfrak{R}_{1212}$ are also known as Riemann symbols of the first and second kind, respectively. Notice that Riemann symbols of the second kind will satisfy the relation $\mathfrak{R}_{1212}=-\mathfrak{R}_{1221}=-\mathfrak{R}_{2112}=\mathfrak{R}_{2121}$, the well-known property of skew-symmetry with respect to the last two indices. It is useful to be aware of the fact that the Christoffel symbols depend only on the coefficients of the first fundamental form and their derivatives. The same holds true for the mixed Riemann curvature tensor. From this point of view, as long as we can find the coefficients of the first fundamental form of a given distribution and their first and second derivatives, we can uniquely define the corresponding Christoffel symbols and hence mixed Riemann curvature tensors. Thus, the process of computing the covariant Riemann curvature tensor and Gaussian curvature is simplified. When $F=0$, formulas (B) and (C) are trivially similar to formula (A). For example, in formula (C), we may substitute the following equation on the left hand side: 


$$
\frac{D}{E} \Gamma_{11}^{2}=\sqrt{\frac{G}{E}}\left(\frac{-E v}{2 G}\right)=\frac{-E v}{2 \sqrt{E G}} \text { or } \frac{D}{E} \Gamma_{12}^{2}=\sqrt{\frac{G}{E}} \frac{G u}{2 G}=\frac{G u}{2 \sqrt{E G}}
$$

We can immediately calculate the same results as found from formula (A) while formula (D) results in a Riemann representation. In this way, we have supplied some more general alternative methods to compute the Gaussian curvature, including the case when $F \neq 0$.

\section{Curvature of Three Life Span Model}

In this section, we give the needed result of derivation by applying formula (B) and (D) for computing our Gaussian curvature. The process and formulas (B) and (D) are complicated, so we decided to tabulate formulas by units, which yields some advantages. It turns out that it is much easier to check partial results than to check the whole equation. It is also much easier to understand why and how we obtain the final results, or in the event of an error it should be much easier to correct it. In model 1, we will deal with Gamma Families. In model 2, we discuss the Weibull Families. In model 3, density function is of form of Inverse Gaussian families.

Model 1: A random variable $X$ has a gamma distribution if its probability density function is of form

$$
\begin{aligned}
& f(x, u, v)=\frac{(u / v)^{u} x^{u-1} \exp (-x u / v)}{\Gamma(u)}, \quad u>0, \quad v>0, x>0 \\
& \ln l=u(\ln u-\ln v)+(u-1) \ln x-\ln \Gamma(u)-\frac{x u}{v} \\
& \frac{\partial \ln l}{\partial u}=1+(\ln u-\ln v)+\ln x-\psi(u)-\frac{x}{v}, \quad \frac{\partial^{2} \ln l}{\partial u^{2}}=\frac{1}{u}-\psi^{\prime}(u) \\
& E=-E\left(\frac{\partial^{2} \ln l}{\partial u^{2}}\right)=\psi^{\prime}(u)-\frac{1}{u}, \quad E(x)=v, \\
& \frac{\partial^{2} \ln l}{\partial v \partial u}=\frac{-1}{v}+\frac{x}{v^{2}}, \quad F=-E\left(\frac{\partial^{2} \ln l}{\partial v \partial u}\right)=-\left(\frac{-1}{v}+\frac{v}{v^{2}}\right)=0 \\
& \frac{\partial \ln f}{\partial v}=\frac{-u}{v}+\frac{x u}{v^{2}}, \quad \frac{\partial^{2} \ln l}{\partial v^{2}}=\frac{u}{v^{2}}-\frac{2 x u}{v^{3}}, \quad G=-E\left(\frac{\partial^{2} \ln l}{\partial v^{2}}\right)=\frac{u}{v^{2}} \\
& \frac{G_{u}-F_{v}}{\sqrt{E G-F^{2}}}=\frac{v}{\sqrt{u \psi^{\prime}(u)-1}} \frac{1}{v^{2}}=\frac{1}{v \sqrt{u \psi^{\prime}(u)-1}} \\
& \frac{\partial}{\partial u}\left[\frac{G_{u}-F_{v}}{\sqrt{E G-F^{2}}}\right]=\frac{-\left(u \psi^{\prime \prime}(u)+\psi^{\prime}(u)\right)}{2 v\left(u \psi^{\prime}(u)-1\right)^{3 / 2}}
\end{aligned}
$$

Now, the information unit needed to apply the formula (B) has been available. In Table 1, we tabulate the most important coefficient of the first fundamental form and their derivatives. It should be aware that $F, F_{u}$ and $F_{v}$ are equal zero. Using one of the fundamental properties of determinants we know that three by three's determinant is zero. This will greatly improve the efficiency of our computation process.

In Table 2, we tabulate what is needed of partial results in formula (B). Also due to the fact that $F_{u}-E_{v}=0$, hence the second term in bracket can also be ignored. Thus, there is only one term needed to compute, and we do so as follows.

$$
\begin{aligned}
& -\frac{1}{2 \sqrt{E G-F^{2}}} \frac{\partial}{\partial u} \frac{G u-F v}{\sqrt{E G-F^{2}}}=-\frac{1}{2} \frac{v}{\sqrt{u \psi^{\prime}(u)-1}}\left[\frac{-\left(u \psi^{\prime \prime}(u)+\psi^{\prime}(u)\right)}{2 v\left(u \psi^{\prime}(u)-1\right)^{3 / 2}}\right] \\
& K_{\text {gатmа }}=\frac{u \psi^{\prime \prime}(u)+\psi^{\prime}(u)}{4\left(u \psi^{\prime}(u)-1\right)^{2}}
\end{aligned}
$$

Notice that the detailed results of six Christoffel symbols are given in summary Table 3. Finally, we list that the formula (D) required results of symbol of Riemann and Gaussian curvature in Table 4. Be awe that 
Table 1. List computing results of coefficient of the first fundamental form and their derivatives.

\begin{tabular}{|c|c|c|c|c|c|c|c|c|c|}
\hline & $E$ & $F$ & $G$ & $E_{u}$ & $F_{u}$ & $G_{u}$ & $E_{v}$ & $F_{v}$ & $G_{v}$ \\
\hline Gamma & $\psi^{\prime}(u)-\frac{1}{u}$ & 0 & $\frac{u}{v^{2}}$ & $\psi^{\prime \prime}(u)+\frac{1}{u^{2}}$ & 0 & $\frac{1}{v^{2}}$ & 0 & 0 & $\frac{-2 u}{v^{3}}$ \\
\hline Weibull & $\frac{C_{1}}{u^{2}}$ & $\frac{C_{2}}{v}$ & $\frac{u^{2}}{v^{2}}$ & $\frac{-2 C_{1}}{u^{3}}$ & 0 & $\frac{2 u}{v^{2}}$ & 0 & $\frac{-C_{2}}{v^{2}}$ & $\frac{-2 u^{2}}{v^{3}}$ \\
\hline $\begin{array}{l}\text { Inverse } \\
\text { Gaussian }\end{array}$ & $\frac{v}{u^{3}}$ & 0 & $\frac{1}{2 v^{2}}$ & $\frac{-3 v}{u^{4}}$ & 0 & 0 & $\frac{1}{u^{3}}$ & 0 & $\frac{-1}{v^{3}}$ \\
\hline
\end{tabular}

Table 2. List important partial results of computing formula.

\begin{tabular}{ccccccc}
\hline & $\Delta$ : Determinant of formula A & $E G-F^{2}$ & $\sqrt{E G-F^{2}}$ & $\frac{1}{\sqrt{E G-F^{2}}}$ & $G_{u}-F_{v}$ & $F_{u}-E_{v}$ \\
\hline Gamma & 0 & $\frac{u \psi^{\prime}(u)-1}{v^{2}}$ & $\frac{\sqrt{u \psi^{\prime}(u)-1}}{v}$ & $\frac{v}{\sqrt{u \psi^{\prime}(u)-1}}$ & $\frac{1}{v^{2}}$ & 0 \\
Weibull & 0 & $\frac{C_{3}}{v^{2}}$ & $\frac{\sqrt{C_{3}}}{v}$ & $\frac{v}{\sqrt{C_{3}}}$ & $\frac{2 u+C_{2}}{v^{2}}$ & \\
Inverse Gaussian & 0 & $\frac{1}{2 u^{3} v}$ & $\frac{1}{u \sqrt{2 u v}}$ & $u \sqrt{2 u v}$ & 0 & $\frac{-1}{u^{3}}$ \\
\hline
\end{tabular}

Where $C_{3}=C_{1}-C_{2}^{2}=1.64493406$.

Table 3. List the computed results of the six Christoffel symbols.

\begin{tabular}{ccccccc}
\hline & $\Gamma_{11}^{1}$ & $\Gamma_{11}^{2}$ & $\Gamma_{12}^{1}$ & $\Gamma_{12}^{2}$ & $\Gamma_{22}^{1}$ & $\Gamma_{22}^{2}$ \\
\hline Gamma & $\frac{u \psi^{\prime \prime}(u)+u^{-1}}{2\left(u \psi^{\prime}(u)-1\right)}$ & 0 & 0 & $\frac{\psi^{\prime}(u)-u^{-1}}{2\left(u \psi^{\prime}(u)-1\right)}$ & $\frac{-u}{2 v^{2}\left(u \psi^{\prime}(u)-1\right)}$ & $\frac{-1}{v}$ \\
Weibull & $\frac{-C_{1}}{C_{3} u}$ & $\frac{C_{1} C_{2} v}{C_{3} u^{3}}$ & $\frac{-C_{2} u}{C_{3} v}$ & $\frac{C_{1}}{C_{3} u}$ & $\frac{-u^{3}}{C_{3} v^{2}}$ & $\frac{C_{2} u-C_{3}}{C_{3}}$ \\
Inverse Gaussian & $\frac{-3}{2 u}$ & $\frac{-v^{2}}{u^{3}}$ & $\frac{1}{2 v}$ & 0 & 0 & $\frac{-1}{v}$
\end{tabular}

Table 4. List the computed results of symbol of Riemann and Gaussian curvature.

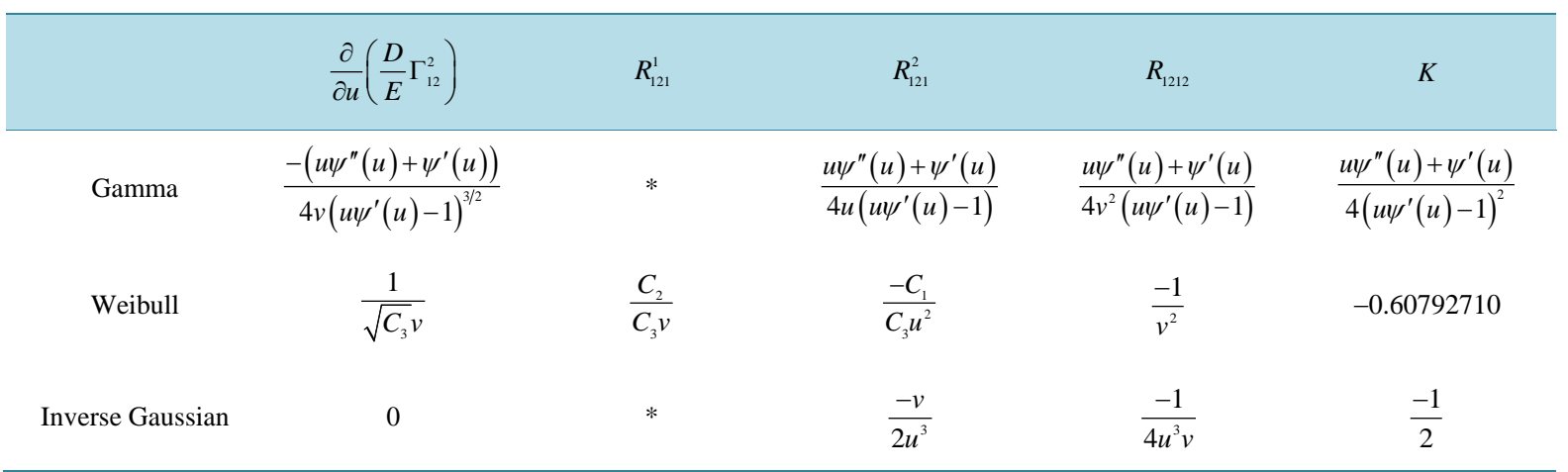




$$
\begin{aligned}
\Gamma_{11}^{2}= & \Gamma_{12}^{1}=0 \\
\mathfrak{R}_{121}^{2}= & \frac{\partial}{\partial v} \Gamma_{11}^{2}-\frac{\partial}{\partial u} \Gamma_{21}^{2}+\sum_{m=1}^{2}\left(\Gamma_{11}^{m} \Gamma_{m 2}^{2}-\Gamma_{21}^{m} \Gamma_{m 1}^{2}\right)=-\frac{\partial}{\partial u} \Gamma_{21}^{2}+\Gamma_{11}^{1} \Gamma_{12}^{2}-\Gamma_{12}^{2} \Gamma_{12}^{2} \\
= & \frac{-\partial}{\partial u}\left[\frac{\psi^{\prime}(u)-u^{-1}}{2\left(u \psi^{\prime}(u)-1\right)}\right]+\left[\frac{u \psi^{\prime \prime}(u)+u^{-1}}{2\left(u \psi^{\prime}(u)-1\right)} * \frac{\psi^{\prime}(u)-u^{-1}}{2\left(u \psi^{\prime}(u)-1\right)}\right]-\left[\frac{\psi^{\prime}(u)-u^{-1}}{2\left(u \psi^{\prime}(u)-1\right)} * \frac{\psi^{\prime}(u)-u^{-1}}{2\left(u \psi^{\prime}(u)-1\right)}\right] \\
& =\frac{\psi^{\prime}(u) u^{-1}+\psi^{\prime \prime}(u)}{4\left(u \psi^{\prime}(u)-1\right)} \\
\mathfrak{R}_{1212} & =\sum_{m=1}^{2} \mathfrak{R}_{121}^{m} g_{m 2}=\mathfrak{R}_{121}^{1} g_{12}+\mathfrak{R}_{121}^{2} g_{22}=\mathfrak{R}_{121}^{2} G=\frac{\psi^{\prime}(u) u^{-1}+\psi^{\prime \prime}(u)}{4\left(u \psi^{\prime}(u)-1\right)} * \frac{u}{v^{2}}=\frac{\psi^{\prime}(u)+u \psi^{\prime \prime}(u)}{4 v^{2}\left(u \psi^{\prime}(u)-1\right)} \\
K= & \frac{\mathfrak{R}_{1212}}{E G-F^{2}}=\left[\frac{v^{2}}{u \psi^{\prime}(u)-1}\right]\left[\frac{\psi^{\prime}(u)+u \psi^{\prime \prime}(u)}{4 v^{2}\left(u \psi^{\prime}(u)-1\right)}\right]=\frac{\psi^{\prime}(u)+u \psi^{\prime \prime}(u)}{4\left(u \psi^{\prime}(u)-1\right)^{2}}
\end{aligned}
$$

Model 2: A random variable $X$ has a Weibull Distribution if its probability density function is of form

$$
f(x, u, v)=\frac{u x^{u-1} \exp \left(-\left(\frac{x}{v}\right)^{u}\right)}{v^{u}}, \quad x>0
$$

$v$ : scale parameter, $u$ : shape parameter

$$
\begin{aligned}
& \ln l=\ln u+(u-1) \ln x-u \ln v-\left(\frac{x}{v}\right)^{u} \\
& \frac{\partial \ln l}{\partial u}=\frac{1}{u}+\ln x-\ln v-\left(\frac{x}{v}\right)^{u} \ln \left(\frac{x}{v}\right), \quad \frac{\partial^{2} \ln l}{\partial u^{2}}=\frac{-1}{u^{2}}-\left(\ln \frac{x}{v}\right)^{2}\left(\frac{x}{v}\right)^{u}, \\
& E=-E\left(\frac{\partial^{2} \ln l}{\partial u^{2}}\right)=\frac{1}{u^{2}}+E\left[\left(\ln \frac{x}{v}\right)^{2}\left(\frac{x}{v}\right)^{u}\right]=\frac{1}{u^{2}}+\frac{\Gamma_{(2)}^{(2)}}{u^{2}}=\frac{\Gamma_{(2)}^{(2)}+1}{u^{2}}=\frac{C_{1}}{u^{2}} \\
& \frac{\partial^{2} \ln l}{\partial v \partial u}=\frac{-1}{v}-\left[\frac{-1}{v}\left(\frac{x}{v}\right)^{u}-\frac{u x}{v^{2}}\left(\frac{x}{v}\right)^{u-1} \ln \frac{x}{v}\right], \\
& F=-E\left(\frac{\partial^{2} \ln l}{\partial v \partial u}\right)=\frac{1}{v}-\frac{1}{v} E\left(\frac{x}{v}\right)^{u}-\frac{u}{v} E\left[\left(\frac{x}{v}\right)^{u} \ln \frac{x}{v}\right]=-\frac{\Gamma_{(2)}^{(1)}}{v}=\frac{C_{2}}{v} \\
& \frac{\partial \ln l}{\partial v}=\frac{-u}{v}+\frac{u x^{u}}{v^{u+1}}, \quad \frac{\partial^{2} \ln l}{\partial v^{2}}=\frac{u}{v^{2}}-\frac{u(u+1) x^{u}}{v^{u+2}}, \quad G=-E\left(\frac{\partial^{2} \ln l}{\partial v^{2}}\right)=\left(\frac{u}{v}\right)^{2} \\
& \psi(2)=\psi(1)+1=-0.5772156649+1=0.422784335=\Gamma^{\prime}(2) \\
& \Gamma^{(2)}(2)=\psi^{\prime}(2)+\left(\Gamma^{\prime}(2)\right)^{2}=0.644934067+0.422784335^{2}=0.823680661 \\
& C_{1}=\Gamma^{(2)}(2)+1=1.823680661, \quad C_{2}=-\Gamma^{\prime}(2)=-0.422784335, \\
& \frac{C_{2}}{C_{1}}=-0.231830245, \quad C_{3}=C_{1}-C_{2}^{2}=1.644934067
\end{aligned}
$$

We are ready to apply the formula (B). The first term involves the $3 \times 3$ determinant expansion. From the previous computation we aware that two terms of expansion are zero, i.e. $E_{v}=0, F_{u}=0$. Hence our final expansion has 


$$
E_{u} F_{v} G-F E_{u} G_{v}-E F_{v} G_{u}=\left[\frac{-2 C_{1}}{u^{3}} \frac{-C_{2}}{v^{2}} \frac{u^{2}}{v^{2}}\right]-\left[\frac{C_{2}}{v} \frac{-2 C_{1}}{u^{3}} \frac{-2 u^{2}}{v^{3}}\right]-\left[\frac{C_{1}}{u^{2}} \frac{-C_{2}}{v^{2}} \frac{2 u}{v^{2}}\right]=\frac{0}{u v^{4}}=0
$$

This means the first term of the determinant can be ignored. Also due to the fact that $E_{v}=0, F_{u}=0$, so the second term in the bracket can also be ignored. Finally, there is only one term that we need to take care of i.e.

$$
\begin{aligned}
K & =-\frac{1}{2 \sqrt{E G-F^{2}}} \frac{\partial}{\partial u}\left(\frac{G_{u}-F_{v}}{\sqrt{E G-F^{2}}}\right)=-\frac{1}{2} \frac{v}{\sqrt{C_{3}}} \frac{2}{\sqrt{C_{3} v}}=\frac{-1}{C_{3}}=\frac{-1}{1.644934067} \\
K & =-0.607927101
\end{aligned}
$$

Using the formula (D) to find the Weibull Distribution Gaussian curvature is our next mission. This is a somewhat messy one, as no short cut can be utilized, since two of the components of Riemann symbols have nonzero values. We show the computation as follows.

$$
\begin{aligned}
\mathfrak{R}_{121}^{1}= & \frac{\partial}{\partial v} \Gamma_{11}^{1}-\frac{\partial}{\partial u} \Gamma_{12}^{1}+\left(\Gamma_{11}^{1} \Gamma_{12}^{1}-\Gamma_{12}^{1} \Gamma_{11}^{1}\right)+\left(\Gamma_{11}^{2} \Gamma_{22}^{1}-\Gamma_{21}^{2} \Gamma_{21}^{1}\right) \\
& =\frac{\partial}{\partial v}\left(\frac{-C_{1}}{C_{3} u}\right)-\frac{\partial}{\partial u}\left(\frac{-C_{2} u}{C_{3} v}\right)+\left(\frac{-C_{1}}{C_{3} u} \frac{-C_{2} u}{C_{3} v}-\frac{-C_{2} u}{C_{3} v} \frac{-C_{1}}{C_{3} u}\right)+\left(\frac{C_{1} C_{2} v}{C_{3} u^{3}} \frac{-u^{3}}{C_{3} v^{2}}-\frac{C_{1}}{C_{3} u} \frac{-C_{2} u}{C_{3} v}\right) \\
& =\frac{C_{2} C_{3}-C_{1} C_{2}+C_{1} C_{2}}{C_{3}^{2} v}=\frac{C_{2}}{C_{3} v} \\
\mathfrak{R}_{121}^{2} & =\frac{\partial}{\partial v} \Gamma_{11}^{2}-\frac{\partial}{\partial u} \Gamma_{12}^{2}+\left(\Gamma_{11}^{1} \Gamma_{12}^{2}-\Gamma_{12}^{1} \Gamma_{11}^{2}\right)+\left(\Gamma_{11}^{2} \Gamma_{22}^{2}-\Gamma_{12}^{2} \Gamma_{21}^{2}\right) \\
& =\frac{\partial}{\partial v}\left(\frac{C_{1} C_{2} v}{C_{3} u^{3}}\right)-\frac{\partial}{\partial u}\left(\frac{C_{1}}{C_{3} u}\right)+\left(\frac{-C_{1}}{C_{3} u} \frac{C_{1}}{C_{3} u}+\frac{C_{2} u}{C_{3} v} \frac{C_{1} C_{2} v}{C_{3} u^{3}}\right)+\left(\frac{C_{1} C_{2} v}{C_{3} u^{3}} \frac{u C_{2}-C_{3}}{C_{3} v}-\frac{C_{1}}{C_{3} u} \frac{C_{1}}{C_{3} u}\right) \\
& =\frac{C_{1}\left(C_{1}-C_{2}^{2}\right)-2 C_{1}^{2}+2 C_{1} C_{2}^{2}}{C_{3}^{2} u^{2}}=\frac{-C_{1}}{C_{3} u^{2}} \\
\mathfrak{R}_{1212} & =\sum_{m=1}^{2} \Re_{121}^{m} g_{m 2}=\mathfrak{R}_{121}^{1} g_{12}+\mathfrak{R}_{121}^{2} g_{22}=\frac{C_{2}}{C_{3} v} \frac{C_{2}}{v}-\frac{C_{1}}{C_{3} u^{2}} \frac{u^{2}}{v^{2}}=\frac{-\left(C_{1}-C_{2}^{2}\right)}{C_{3} v^{2}}=\frac{-1}{v^{2}} \\
K= & \frac{\Re_{1212}}{E G-F^{2}}=\frac{-1 / v^{2}}{C_{3} / v^{2}}=-\frac{1}{C_{3}}=-0.607927101
\end{aligned}
$$

Model 3: A random variable $X$ has an Inverse Gaussian Distribution if its probability density function is of form

$$
\begin{aligned}
& f(x, u, v)=\left(\frac{v}{2 \pi x^{3}}\right)^{\frac{1}{2}} \exp \left(-\frac{v}{2 u^{2} x}(x-u)^{2}\right), \quad x>0 \\
& \ln l(x, u, v)=\frac{1}{2}\left(\ln v-\ln 2 \pi x^{3}\right)-\frac{v}{2 u^{2} x}(x-u)^{2}, \\
& \frac{\partial \ln l}{\partial u}=\frac{v\left(u^{2}(x-u)+u(x-u)^{2}\right)}{x u^{4}}=\frac{v(x-u)}{u^{3}}, \quad \frac{\partial^{2} \ln l}{\partial u^{2}}=\frac{-v(3 x-2 u)}{u^{4}} \\
& E=-E\left(\frac{\partial^{2} \ln l}{\partial u^{2}}\right)=\frac{v}{u^{3}}, \quad E(x)=u, \quad \operatorname{var}(x)=\frac{u^{3}}{v}, \\
& \frac{\partial^{2} \ln l}{\partial v \partial u}=\frac{x-u}{u^{3}}, \quad F=-E\left(\frac{\partial^{2} \ln l}{\partial v^{2}}\right)=-\frac{u-u}{u^{3}}=0 \\
& \frac{\partial \ln f}{\partial v}=\frac{1}{2 v}-\frac{(x-u)^{2}}{2 u^{2} x}, \quad \frac{\partial^{2} \ln l}{\partial v^{2}}=\frac{-1}{2 v^{2}}, \quad G=-E\left(\frac{\partial^{2} \ln l}{\partial v^{2}}\right)=\frac{1}{2 v^{2}}
\end{aligned}
$$


Again, the information unit needed to apply the formula (B) has been available. Again, we aware that $F, F_{u}$ and $F_{v}$ are all equal zero. Hence the first term of determinant is zero. Also due to the fact that $G_{u}-F_{v}=0$ hence the first term in bracket can also be ignored. There is only one term need to compute and we do it as follows.

$$
\begin{aligned}
& -\frac{1}{2 \sqrt{E G-F^{2}}}\left[-\frac{\partial}{\partial v} \frac{F u-E v}{\sqrt{E G-F^{2}}}\right]=-\frac{1}{2} \sqrt{2 u^{3} v}\left[\frac{1}{2} \sqrt{\frac{2}{u^{3} v}}\right]=\frac{-1}{2} \\
& K_{I G}=\frac{-1}{2}
\end{aligned}
$$

Notice that the detailed results of six Christoffel symbols are given in summary table. Next, we apply the formula (D) to compute the curvature as below.

$$
\begin{aligned}
\mathfrak{R}_{121}^{2} & =\frac{\partial}{\partial v} \Gamma_{11}^{2}-\frac{\partial}{\partial u} \Gamma_{12}^{2}+\left(\Gamma_{11}^{1} \Gamma_{12}^{2}-\Gamma_{12}^{1} \Gamma_{11}^{2}\right)+\left(\Gamma_{11}^{2} \Gamma_{22}^{2}-\Gamma_{12}^{2} \Gamma_{21}^{2}\right) \\
& =\frac{\partial}{\partial v}\left(\frac{-v^{2}}{u^{3}}\right)+\frac{v^{2}}{2 v u^{3}}+\frac{v^{2}}{v u^{3}}=\frac{-v}{2 u^{3}} \\
\mathfrak{R}_{1212} & =\sum_{m=1}^{2} \Re_{121}^{m} g_{m 2}=\mathfrak{R}_{121}^{1} g_{12}+\mathfrak{R}_{121}^{2} g_{22}=\mathfrak{R}_{121}^{2} G=\frac{-1}{4 u^{3} v} \\
K & =\frac{\Re_{1212}}{E G-F^{2}}=\frac{-1}{4 u^{3} v}\left(2 u^{3} v\right)=\frac{-1}{2}
\end{aligned}
$$

To summarize and compare the Gaussian curvature computed in Equations (3.1), (3.2), (3.3), (3.4), (3.5), and (3.6), it is obvious that formula (B) and (D) give us the identical results.

\section{Concluding Remark}

It is also a well-known fact that two surfaces which have the same Gaussian curvature are always isometric and bending invariant. For instance, Struik, D.J. on p. 120 provided an excellent example that demonstrated a correspondence between the points of a catenoid and that of a right helicoid, such that at corresponding points, the coefficients of the first fundamental form and the Gaussian curvatures are dentical. In fact, one surface can pass into the other by a continuous bending. This has been demonstrated by the deformation of six different stages. However, if the Gaussian curvature is different, the two surfaces will not be isometric. For example, a sphere and plane are not locally isometric because the Gaussian curvature of a sphere is nonzero while the Gaussian curvature of a plane is zero. This is why any map of a portion of the earth must distort distances. One of the most important theorems of the $19^{\text {th }}$ century is "Theorema Egregium". Many mathematicians at the end of the $18^{\text {th }}$ century, including Euler and Monge, had used the Gaussian curvature, but only when defined as the product of the principal curvatures. Since each principal curvature of a surface depends on the particular way where the surface is defined in $R^{3}$, there is no obvious reason for the product of the principal curvatures to be intrinsic to that particular surface. Gauss published in 1827 that the product of the principal curvatures depends only on the intrinsic geometry of the surface revolutionized differential geometry. Gauss wrote " "The Gaussian curvature of a surface is a bending invariant', 'a most excellent theorem', 'This is a Theorema egregium'”. In this theorem, Gauss proved that the Gaussian curvature, $K$, of a surface, depends only on the coefficient of the first fundamental form and their first and second derivatives. This important geometric fact will link the concepts of bending and isometric mapping.

\section{References}

[1] Kass, R.E. and Vos, P.W. (1997) Geometrical Foundations of Asymptotic Inference. John Wiley \& Sons, Inc., New York. http://dx.doi.org/10.1002/9781118165980

[2] Kass, R.E. (1989) The Geometry of Asymptotic Inference (with Discussion). Statistical Science, 4, 188-234.

[3] Struik, D.J. (1961) Lectures on Classical Differential Geometry. 2nd Edition, Dover Publications, Inc., New York.

[4] Lawless, J.F. (1982) Statistical Models and Methods for Lifetime Data. John Wiley \& Sons, Hoboken. 
[5] Chen, W.W.S. (1980) On the Tests of Separate Families of Hypotheses with Small Sample Size. Journal of Statistical Computation and Simulation, 2, 183-187. http://dx.doi.org/10.1080/00949658008810406

[6] Chen, W.W.S. (1982) Simulation on Probability Points for Testing of Lognormal or Weibull Distribution with a Small Sample. Journal of Statistical Computation and Simulation, 15, 201-210. http://dx.doi.org/10.1080/00949658208810583

[7] Chen, W.W.S. (1987) Testing Gamma and Weibull Distribution: A Comparative Study. Estadistica, 39, 1-26.

[8] Chen, W.W.S. (1983) Testing Lognormal and Exponential Distributions: Estimation of Percentile Points. American Journal of Mathematical and Management Sciences, 3, 165-196. http://dx.doi.org/10.1080/01966324.1983.10737123

[9] Gupta, A.K. (1952) Estimation of the Mean and Standard Deviation of a Normal Population from a Censored Sample. Biometrika, 39, 260-273. http://dx.doi.org/10.1093/biomet/39.3-4.260

[10] Balakrishnan, N. and Chen, W.W.S. (1999) Handbook of Tables for Order Statistics from Lognormal Distributions with Applications. Kluwer Academic Publishers, Norwell. http://dx.doi.org/10.1007/978-1-4615-5309-0

[11] Balakrishnan, N. and Chen, W.W.S. (1997) CRC Handbook of Tables for Order Statistics from Inverse Gaussian Distributions with Applications. CRC Press, Boca Raton.

[12] Gray, A. (1993) Modern Differential Geometry of Curves and Surfaces. CRC Press, Inc., Boca Raton. 


\section{Appendix}

Next, we define the six well known Christoffel symbols see Struik, D.J. or Gray A. [12]

$$
\begin{aligned}
& \Gamma_{11}^{1}=\frac{G E_{u}-2 F F_{u}+F E_{v}}{2\left(E G-F^{2}\right)}, \quad \Gamma_{12}^{2}=\frac{E G_{u}-F E_{v}}{2\left(E G-F^{2}\right)}, \quad \Gamma_{11}^{2}=\frac{2 E F_{u}-E E_{v}-F E_{u}}{2\left(E G-F^{2}\right)}, \\
& \Gamma_{22}^{1}=\frac{2 G F_{v}-G G_{u}-F G_{v}}{2\left(E G-F^{2}\right)}, \quad \Gamma_{12}^{1}=\frac{G E_{v}-F G_{u}}{2\left(E G-F^{2}\right)}, \quad \Gamma_{22}^{2}=\frac{E G_{v}-2 F F_{v}+F G_{u}}{2\left(E G-F^{2}\right)}
\end{aligned}
$$

we applied the following integral results

$$
\begin{gathered}
E\left(\left(\ln \left(\frac{x}{v}\right)\right)^{2}\left(\frac{x}{v}\right)^{u}\right)=\int_{0}^{\infty}\left(\ln \frac{x}{v}\right)^{2}\left(\frac{x}{v}\right)^{u} \frac{u x^{u-1}}{v^{u}} \mathrm{e}^{-\left(\frac{x}{v}\right)^{u}} \mathrm{~d} x=\frac{\Gamma^{(2)}(2)}{u^{2}} ; \\
E\left(\left(\ln \left(\frac{x}{v}\right)\right)\left(\frac{x}{v}\right)^{u}\right)=\int_{0}^{\infty}\left(\ln \frac{x}{v}\right)\left(\frac{x}{v}\right)^{u} \frac{u x^{u-1}}{v^{u}} \mathrm{e}^{-\left(\frac{x}{v}\right)^{u}} \mathrm{~d} x=\frac{\Gamma^{\prime}(2)}{u}
\end{gathered}
$$

we define the nth derivative of the gamma function:

$$
\Gamma^{(n)}(x)=\int_{0}^{\infty} \mathrm{e}^{-t} t^{x-1}(\ln t)^{n} \mathrm{~d} t, \quad x>0
$$


Scientific Research Publishing (SCIRP) is one of the largest Open Access journal publishers. It is currently publishing more than 200 open access, online, peer-reviewed journals covering a wide range of academic disciplines. SCIRP serves the worldwide academic communities and contributes to the progress and application of science with its publication.

Other selected journals from SCIRP are listed as below. Submit your manuscript to us via either submit@scirp.org or Online Submission Portal.
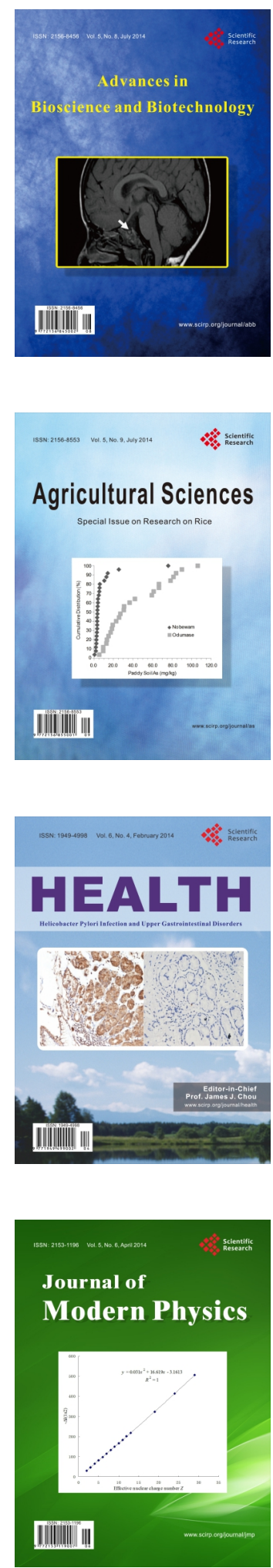
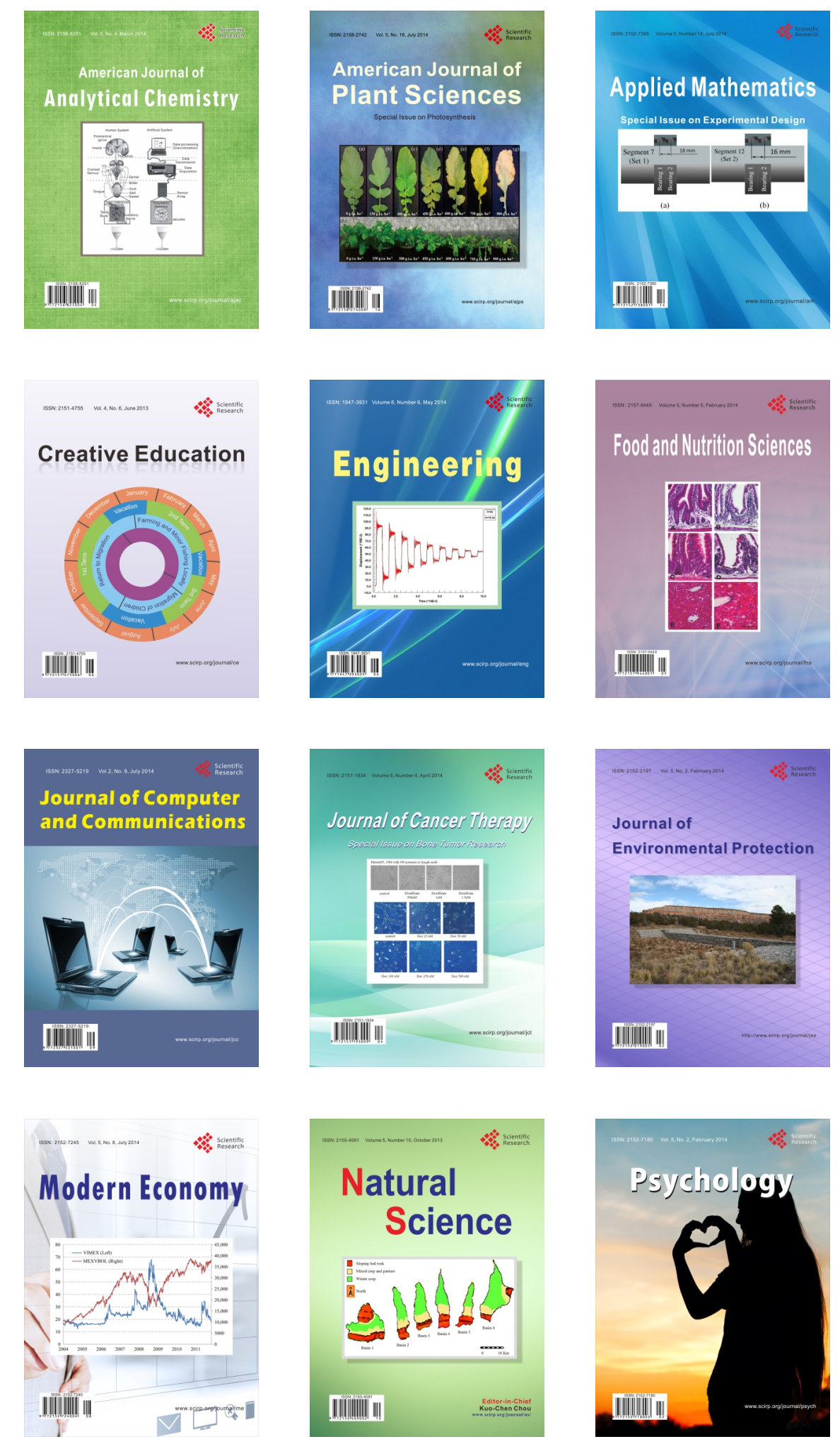